\title{
A Possible Merge of FRET and SPR Sensing System for Highly Accurate and Selective Immunosensing
}

\author{
Jaebeom Lee, ${ }^{*}$ Hongxia Chen, ${ }^{\dagger}$ Jaewook Lee, Fangfang Sun, Cheol-Min Kim, ${ }^{\ddagger}$ Chulhun L. Chang, ${ }^{\ddagger}$ and Kwangnak Koh
}

\author{
Department of Nanomedical Engineering, Pusan National Lniversity, Airvang 627-706, Korea \\ ${ }^{\star E}$-mail: jaebeomápusan ac.kn \\ ${ }^{\dagger}$ College of Life Science, Shanghai Lninersity, Shanghai 2004t4, China \\ -School of Medicine, Pusan National Cniversity. Busan 602-739, Korea \\ Received September 1. 2009. Accepted September 22. 2009
}

\begin{abstract}
Immuno-sensing for high accurate and selective sensing was performed by fluorescence spectroscopy and surface plasmon resonance (SPR), respectively. Engineered assembly of two fluorescent quantum dots (QDs) with bovine serum albumin (BSA) and anti-BSA was fabricated in PBS buffer for fluorescence analysis of fluorescence resonance energy transfer (FRET). Furthennore, the same bio-moieties were immobilized on Au plates for SPR analysis. Natulrally-driven binding affinity of inmuno-moieties induced FRET and plasmon resonance angle shift in the nanoscale sensing system. Interestingly, the sensing ranges were uniquely different in two systems: e.g., SPR spectroscopy was suitable for highly accurate analysis to measure in the range of $10^{-15-10} \mathrm{ng} / \mathrm{mL}$ while the QD fluorescent sensing system was relatively lower sensing ranges in $10^{10 \cdots \cdot} \mathrm{ng} / \mathrm{mL}$. However, the QD sensing system was larger than the SPR sensing system in terms of sensing capacity per one specimen. It is, therefore, suggested that a mutual assistance of FRET and SPR combined sensing sy stem would be a potentially promising candidate for high accuracy and reliable in situ sensing sy stem of inmune-related diseases.
\end{abstract}

Key Words: Immunosensor. FRET. SPR. Sensing șystem. Nanoparticle

\section{Introduction}

Immunosensors paid special attention on device design and fabrication because high characteristics of selectivity and sensitivity of biological moieties can be easily adopted. compared with any other electrochemical or phy sical sensing sy stem. ${ }^{1.5}$ However noticeable signal losses in the ș'stem may be potential limits to fabricate highly sensitive sensing. ${ }^{6 \&}$ Mostly, the losses result in the transferring failure of the signal carriers such as photons or electrons in many different interfaces. In particular. the interfaces of biomaterials and transducers are significantly crucial not only where the biological sensing moieties are located to collect the firstly monitored signals, but also where viability of biologically sensing materials on the surface of the fabricated transducers should be maintained during the sensing process. So, the transducer is the most important controlling part and is easy to be a bottleneck for fabricating high accurate and selective sensing device. ${ }^{9.13}$ Since the biological moieties are utilized for sensing devices. two regimes. biological stability (or bioinertness). and high selectivity and sensitivity are most-carefully handled factors for developing sustainable device. The transducer parts are. in general. composed of bio-inert. bio-compatible. and highly signal-transferable materials to convince two main factors as the above described. To gain the biological selectivity and sensitivity, a pair of antigen (aG)-antibody $(a B)$ is one of unique candidates for sensing process. However the immobilization of immuno-materials requires well-controlled surface chemistry techniques. For these reasons, nanotechnology is quite promising to improve high signal transferability of bio-compatible surface. Moreover, minimized contact areas with biological moieties on the transducers may retard undesirable transfor- mation of bio-moieties during sensing process.

When the sensing systems are miniaturized on the benefit of nanotechnology, sensitivity and selectivity may be highly increased. However, the sensing capacity, i.e.. concentration or molarity of target materials would be considerable issues since it would approach easily to certain saturated level quantitatively, in another word. upper detection limit. where it is not possible to acquire distinguishable signals. For example, in an extreme environment such as warfare. or off-shore, each personnel brings individual personal chem/bio sensing kits that require high sensitivity as well as probably high sensing capacity of targeting materials because of harsh ground situation. ${ }^{\text {t.13 }}$ However. it would be a controversy to install two crucial characteristics in a nanoscale devices because high capacity of the selected sample generally requires pre-treatment for analysis and shows relatively lower selectivity and sensitivity because the sensing analy tes would have many different obstacles statistically. Therefore. we may need other approaches to overwhelnt current conplicated problems. Here, we present a potential means wia the consolation of two different sensing tecluniques, i.e., surface plasmon resonance (SPR) spectroscopy. and fluorescence resonance energy: transfer (FRET)-based photoluminescence (PL) spectroscopy. (Figure 1) One reason we select these two techniques for one set of sensing devices would be result from distinguishably different sensing ranges - one for $\mathrm{pg} / \mathrm{nL}$ to $\mathrm{ng} / \mathrm{mL}$ level and another for $\mathrm{ng} / \mathrm{mL}$ to $\mu \mathrm{g} / \mathrm{mL}$ level - at which each sensing device presents optimum working condition. Highly sensitive devices would require long processing time. ligh cost of equipments and their maintenance, and skilled techniques. Another reason of the combination of these two sensors is the considerable difference of sample quantity requirement for detection. SPR sensor needs 
(a)

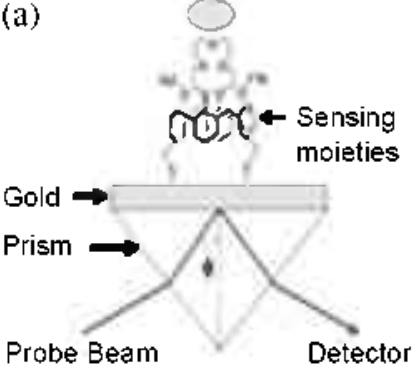

(b)

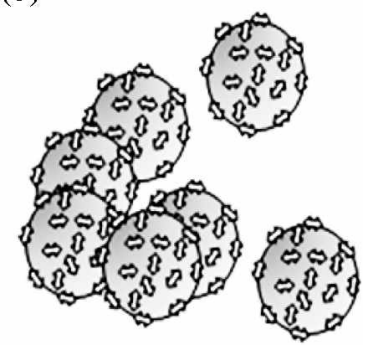

(c)

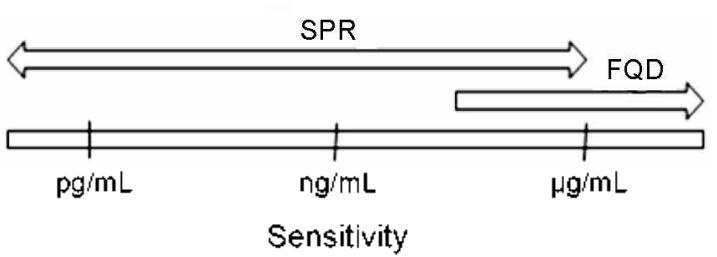

Figure 1. a schematic drawing of two different nanoscale Immunosensors; (a) surface plasmon resonance ( $\mathrm{SPR}$ ) spectroscopy, (b) fluorescence resonance energy transfer (FRE l")-based photoluminescence (PL) spectroscopy, and (c) their sensitivity ranges.

very little amount of samples up to $\mu g$ level per a series of representative experiments with repetition. Meanwhile. FRET using nanoparticles may require. in general, relatively higher concentration than that of SPR sensors to increase signal/noise ratio even though it is related to the detection limit of spectrophotometers as well as the bioconjugation techniques of nanoparticles. The combination of two different aspects of sensing device would have cost-effective and time-saving merits to monitor pathogenic materials. It is. therefore. suggested that FRET and SPR combined sensing system would be in situ multi-ranged sensing devices for immune-related diseases.

\section{Experimental Detail}

Chemicals. BSA. anti-BSA. ovalbumin (OVA), N-hydroxysuccinimide (EDC) $N$-ethỵl-1/7-(dimethỵ laminopropyll) carbodiimide hydrochloride (NHS) and phosphate-buffered saline (PBS) were obtained from Sigma Chemical Co. (St. Louis. MO. USA). $\mathrm{Al}_{2} \mathrm{Te}_{3}$ was purchased from CERAC Inc (Milwaukee. USA). All other reagents including $\mathrm{Cd}\left(\mathrm{ClO}_{4}\right)_{2} \cdot \mathrm{H}_{2} \mathrm{O}, \mathrm{H}_{2} \mathrm{SO}_{4}$ were purchased from Aldrich Chemical Co. (Milwaukee. WI. USA). Prolinker ${ }^{\mathrm{TM}}$ as surface linkage material was purchased from Proteogen Co. (Seoul. Korea). A Milli-Q grade (> $18.2 \mathrm{~m} \Omega \cdot \mathrm{cm}$ ) water was used for the preparation of buffer solutions and NP synthesis.

Quantum dot based FRET sensor. For preparation of fluorescence resonance energy transfer (FRET) sensor. aqueous dispersions of green and yellow fluorescent quantum dots were prepared at the peak wavelength of $542 \mathrm{~nm}$ and $646 \mathrm{~nm}$. respectively, according to the Weller's Method. ${ }^{16}$ EDC and NHS were used for bioconjugation of NPs and bio-moiety through the formation of an oxime bond from an amine group and a carboxy lic group. "Briefly, the mixture containing $0.015 \mathrm{mM}$ of $\mathrm{CdTe}$ QD5. 2.5 mM NHS and 0.2 M EDC in $\mathrm{pH} 7.4$ PBS buffer solutions were prepared and stirned for $30 \mathrm{~min}$ in room temperature. respectively. For the demonstration of FRET sensors in microrange of antigen-antibody solutions. each $5 \mu \mathrm{g} / \mathrm{mL}$ of BSA and anti-BSA was added into the respective mixture solution containing CdTe QDs. Then, the solutions were stirred for $2 \mathrm{~h}$ at room temperature. As a result. each different batch solution. i.e.. CdTe $(6+6 \mathrm{~nm}) / \mathrm{BSA}$, and CdTe $(5+2 \mathrm{~nm}) / \mathrm{anti}-\mathrm{BSA}$ were conjugated. The conjugation was confirmed in the measurement of global size change and optical properties of nanocontex by zeta-sizer and spectroscopic method.

Au-nanoplate based SPR sensor. The same $\mathrm{aG}-\mathrm{aB}$ solution was used for SPR sensing experiments. A detailed experimental procedure is as follows: A microscope cover glass $(18 \times 18 \times$ $0.15 \mathrm{~mm}$, with a refractive index of 1.515. Matsunani, Japan) with gold laser was used as a substrate for the formation of ProLinker ${ }^{\mathrm{TM}}$ self assembled monolayer (SAM). The gold film (thickness $\approx 50 \mathrm{~nm}$ ) was deposited on the cover glass by the sputter coating sy'stem (E5000. Polaron Co.. U.K.) under conditions of $2.0 \times 10^{--}$mbar and $20 \mathrm{mLA}$ for $135 \mathrm{~s}$. The sputtered Au substrate was rinsed using distilled water. methanol and acetone. sequentially. Then, the gold chip was dried in a nitrogen stream softly and ready to use. The calix[4]crown solution was prepared by the mixture of chloroform and methanol. Their volume ratio was $1: 100$. The SAM was fonmed by immersion of the gold chip into the $0.1 \mathrm{mM}$ calis[4]crown solution. After immobilization. the sensor chip was rinsed with cliloroform-methanol nixture solution ( $1: 100 . \mathrm{V} v$ ). methanol for $15 \mathrm{~min}$ and then dried under $\mathrm{N}_{2}$ stream. The measurement of molecular interaction between anti-BSA and BSA was investigated by SPR. Anti-BSA can be well oriented onto the self-assembled prolinker layer. Proteins anti-BSA and OVA were inunobilized on the Prolinker ${ }^{\text {TM }}$ SAM. respectively. The anti-BSA solution for the immobilization of probe protein was prepared with $100 \mu \mathrm{g} / \mathrm{nL}$ concentration in PBS solution ( $\mathrm{pH} 7,4)$. For blocking the non-specific binding of BSA onto the site of Prolinker ${ }^{\text {TM }}$ SAM without aB, the chip was treated in $30 \mathrm{mg} / \mathrm{nL}$ OVA in PBS solution ( $\mathrm{pH} 7.4$ ). SPR angle shifts induced by the interaction between anti-BSA and BSA were measured by SPR spectroscopy (SPR $i$ Lab, K-MAC Co. Ltd. Korea). The measured concentration of BSA solution ranged from $5.0 \mathrm{pg} / \mathrm{mL}$ to $10 \mu \mathrm{g} / \mathrm{mL}$ in PBS solution ( $\mathrm{pH} 7.4$ ). The results were averaged after measurement and uniformly smoothed by adjacent averaging.

\section{Results and Discussion}

FRET based immunosensor. FRET is quite unique optical phenomenon when a fluorescent material is in the proximity of the different fluorescent nuterial. The higher bandgap energy was transferred to the lower bandgap energy by dipole-dipole interaction in the range of $10 \mathrm{~nm}$ scale. The sensitivity to distance demonstrates $1 / R^{6}$ of fluorescence energy transfer change. Here. R stands for the distance between two fluorescence materials. Figure 1(a) presents a schentatic demonstration of FRETinduced immunosensing system. $5 \mu \mathrm{g} / \mathrm{mL}$ of BSA and anti-BSA were used to bind CdTe QDs solution with different emissions at 646 and $542 \mathrm{~mm}$. respectively. As soon as each luminescent QDs are bound by aG-aB reaction, the peak intensity at $5+2 \mathrm{~nm}$ was decreased while the emission at 646 mu was sinultaneously increased. The distance $\left(R_{\mathrm{DA}}\right)$ between two fluorescent materials 
(a)
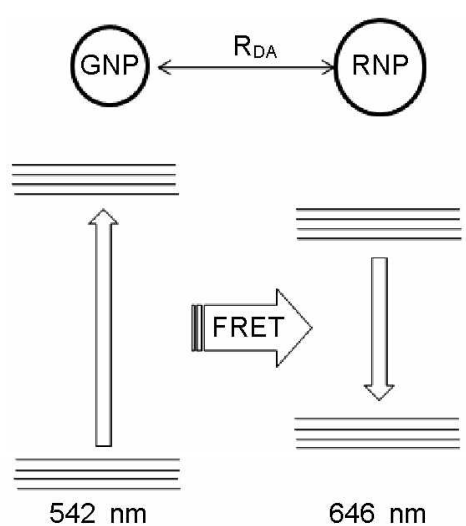

(b)

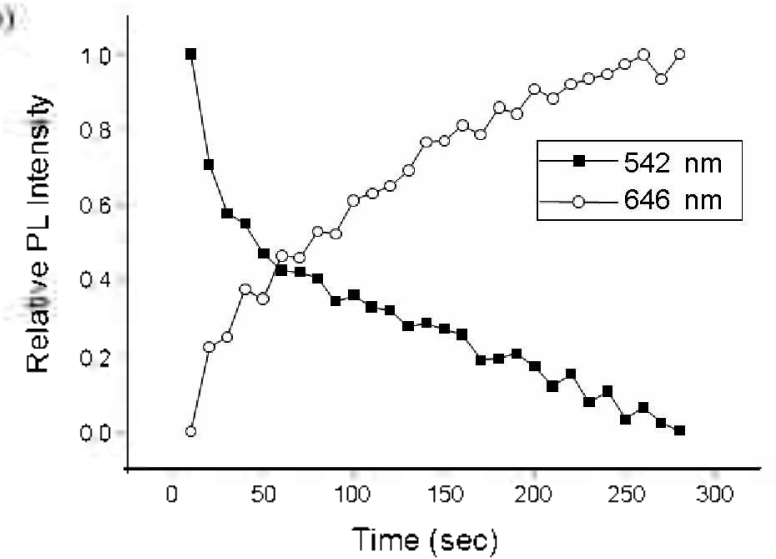

Figure 2. (a) schematic drawing of fluorescence resontance energy transter between green fluorescent nanoparticles (GNP) and red fluorescent nanoparticles ( $\mathrm{RNP}$ ), (b) relative $\mathrm{PL}$ intensity of two different Cdle NPs where both are biologically conpugated with antigen (aG)antibody (aB) of BSA.

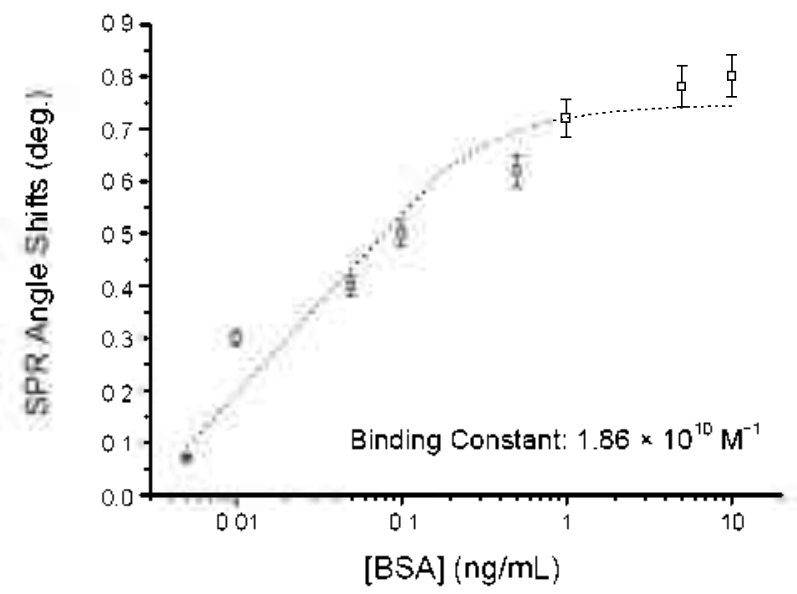

Figure 3. Dose-response curves of anti-BSA immobilized sensor chip represented by SPR angle shifts according to the interaction between anti-BSA and BSA on Prolinker ${ }^{T M}$ SAM. The dotted line indicates the result of one site binding fitting with a binding constant of $1.86 \times$ $10^{10} \mathrm{M}^{1}$

was located at less than $10 \mathrm{rm}$ and the higher bandgap energy was transferred to the lower bandgap energy: The sensitivity of FRET-based sensor can be approximated from the theoretical calculation given in Eq. 1 .

$$
\begin{aligned}
R_{i} & =0.2108\left[J_{\mathrm{DA}}(\lambda) \kappa^{-} n^{-4} \Phi_{\mathrm{D}}\right]^{10} \\
E & =R_{(1}{ }^{6} /\left[R_{\mathrm{G}}^{6}+R_{\mathrm{DA}}{ }^{6}\right]
\end{aligned}
$$

where, $R_{\mathrm{LA}}$ is the distance between the fluorescence donor and acceptor, and $R_{i}$ is the distance $(\dot{A})$ at which half of the energy is transferred (the Forster radius). $J_{\mathrm{DA}}$ in the second equation is the donor/acceptor spectral overlap integral. $\kappa$ is a geometric factor associated with the orientation of the donor and acceptor dipoles. The orientation, $\kappa$ is generally considered as constant when conjugations between two 0 -D fluorophores are carried out.

The relative intensity was shown in Figure l(b) that the $Q D$ green lost its original fluorescence intensity while the QD red gain its intensity. From the comparison of actual intensity changes, the energy transfer was about $27 \%$ from $Q D$ green to $Q D$ red with little loss of energy. Furthenmore. Figure l(b) presents the time-based intensity change when two QDs are conjugated. The $50 \%$ of intensity was changed within $100 \mathrm{sec}$ after the interval of the conjugation of $\mathrm{aG}-\mathrm{aB}$, which is a good candidate for rapid analysis method. Meanwhile the sensitivity of this sensor reached up to the $\mu \mathrm{g}$ range of aG and the selectivity of this sensor would be decided by the quality of the inmumo-reaction as well as the surface chemistry of nanoparticles with biological moieties. The used sample quantity was about $\mu \mathrm{g}$ ranges in the fluorescence spectroscopic experiment. The next sensor we suggest can measure aGs up to pg range that is more sensitive with different sensing mechanism even though we used the same biological moieties for selectivity.

Au-nanoplate based SPR sensor. To confirm the chip's sensitivity. the aB-modified chip was then incubated with 7 different concentrations of antigen solutions ranging from from $5.0 \mathrm{pg} /$ $\mathrm{mL}$ to $10 \mu \mathrm{g} / \mathrm{mL}$ in PBS solution. After rinsing with the PBS buffer. SPR analy'sis was carried out at each concentration. Figure 2 showed the various concentration of BSA that was bound to the corresponding antibody chip. As the BSA concentration increased. the SPR angle of the anti-BSA chip was gradually slifted to the right and the total shift degrees were increased as the concentration of BSA was increased. These data confirmed that the antigen-antibody interaction occurred on the surface of the ProLinker ${ }^{\mathrm{TN}}$ SAM and suggested that the ability to make immunochip on ProLinker ${ }^{\mathrm{TM}}$ surface with high sensitivity. The data was linearly proportional to the range of $\mathrm{pg} / \mathrm{mL} \sim \mathrm{ng} / \mathrm{mL}$. However, the after the $\mathrm{ng} / \mathrm{nL}$ of concentration. the signal was renarkably saturated to draw the plain line where is the maximum sensing concentration. Here. it would be necessary to combine two different nano sensing systems for expanding sensing range from $\mathrm{pg} / \mathrm{mL} \sim \mu \mathrm{g} / \mathrm{mL}$ because actual environmental condition would vary with no expectation. In particular, nilitarypurpose biosensing system would significantly require multiranges of concentration covered sensors with high sensitivity and selectivity.

\section{Conclusion}

Two different nanotechnology-induced biosensors were demonstrated to detect BSA via immuno-activity of anti-BSA by means of FRET and SPR techniques. Since each sensor gives 
different concentration ranges of sensing ability they are quite complementary to embody sensing șistem from $\mu \mathrm{g} / \mathrm{mL}$ to $\mathrm{pg} /$ $\mathrm{mL}$.

Acknowledgments. This study was supported by a grant of the Korea Healthcare technology R\&D Project (A080854). Ministry for Health Welfare \& Family Affairs. Republic of Korea.

\section{References}

1. Lei, C. X.; Gong, F. C.; Shen, G. L.; Yu, R. Q. Sensor Acnat $B$-Chem 2003, 96, 582-588.

2. Chen, H. X; Kim, Y. S.; Keum, S. R.; Kim, S. H.; Choi, H. J.; Lee, J. B.: An, W. G.: Koh, K. Sensors 2007, 7, 1216-1223.

3. Lyon, L. A.; Musick, M. D.; Natan, M. I. .Hal Chem. 1998, $70,5177-5183$

4. Wilson, M. S. Anal Chem. 2005, 77, 1496-1502.

5. Wang, J. Electroanalvsis 2005, 17, 7-14

6. Balasubramanian, K, Burghard, M. Anal Bioanal Chem. 2006, $385,452-468$.
7. Park, I. K.; Yee, H. I.: Lee, K. S.; Lee, W. Y.: Shin, M. C.: Kim, T. H.: Kim, S. R. Anal Chim Acta 1999, 390, 83-91.

8. Gopel, W., Heiduschka, P. Biosens. Bioelectron. 1995, 10, 853883.

9. Tumer, P. F. A.; Karube, I.; Wilson, G. S. Biosensons: Fundamentals and Applications: Oxford University Press: USA, 1987.

10. Eggins, R. B. Chemical Sensors and Biosensors; Wiley: New York, 2002.

11. Svorc, I.; Miertus, S.; Katrlik, J.; Stredanshy, M. Anal. Chem. $1997,69,2086-2090$.

12. R. S. S. Biosens. Bioelection 1994, 9. 243-264.

13. Lee, S. M: Lee, W. Y. Buhl. Korean Chent. Soc. 2002, 23, $1169-$ 1172.

14. Pejcic, B.: De Marco, R:; Parkinson, G. Analyst 2006, 131, $1079-1090$

15. Mark, R. H.: Ebtisam, W. Am. J. Appl. Sci. 2005, 2, 796-805.

16. Gaponik, N.; Talapin, D. V.; Rogach, A. L.; Hoppe, K.; Shevcherko, E. V.; Komowski, A.; Eychmueller, A.; Weller, H. $J$. Phs Chem B 2002, 106, 7177-7185.

17. Lee, J. B.; Govorov, A. O.; Dulka, J.: Kotov, N. A. Nano Lett. $2004,+2323-2330$. 